\title{
Identification of Molecular Flipping of an Asymmetric Tris(phthalocyaninato) Lutetium Triple-Decker Complex by Scanning Tunneling Microscopy/Spectroscopy
}

\author{
Xianghua Kong ${ }^{1}$, Shengbin $\mathrm{Lei}^{2}$, Yanlian $\mathrm{Yang}^{2}$, Ke Deng ${ }^{2}$, Guicun $\mathrm{Qi}^{2}$, and Chen Wang ${ }^{2}(\varangle)$ \\ ${ }^{1}$ Institute of Chemistry, Chinese Academy of Sciences, Beijing 100190, China \\ ${ }^{2}$ National Center for Nanoscience and Technology, Beijing 100190, China \\ Received: 14 December 2008/Revised: 11 January 2009/Accepted: 12 January 2009 \\ (C) Tsinghua University Press and Springer-Verlag 2009. This article is published with open access at Springerlink.com
}

\begin{abstract}
The assembling behavior and electronic properties of asymmetric tris(phthalocyaninato) lutetium tripledecker sandwich complex molecules $\left(\mathrm{Lu}_{2} \mathrm{Pc}_{3}\right)$ on highly oriented pyrolytic graphite (HOPG) surfaces have been studied by scanning tunneling microscopy/spectroscopy (STM/STS) methods. Phase transitions were observed at different bias polarities, involving an ordered packing arrangement with fourfold symmetry at negative bias and an amorphous arrangement at positive bias. Molecular switching behaviour for individual $\mathrm{Lu}_{2} \mathrm{Pc}_{3}$ molecules was reported here according to the bias-polarity-induced flipping phenomena and the peak shift in $\mathrm{d} I / \mathrm{d} V$ versus $V$ curves at different voltage scanning directions. The sensitive response of the strong intrinsic molecular dipole to an external electric field is proposed to be responsible for molecular switching of $\mathrm{Lu}_{2} \mathrm{Pc}_{3}$ at the solid/liquid interface.
\end{abstract}

\section{KEYWORDS}

Triple-decker sandwich complex, phase transition, molecular switch, scanning tunneling microscopy/ spectroscopy

\section{Introduction}

Controlled construction and tuning of molecular nanostructures is a prerequisite for the fabrication of molecular devices and precise control of the molecular orientation and ordering is necessary in order to tailor the physical and chemical properties of molecular architecture. Some external stimuli such as electric fields and thermal annealing can be employed to tune molecular assembly [1-7]. Electric -polarity-driven molecular switching behavior has been the focus of considerable attention [8-10], and in this case the intrinsic dipole of the molecules is suggested to be a crucial issue for molecular switching. It was reported that non-planar tinnaphthalocyanine $(\mathrm{SnNc})$ molecules have strong intrinsic molecular dipoles (1.48 D) which enable SnNc to exhibit voltage-induced flipping on a surface [9]. On the other hand, we have previously presented a strategy of attaching functional groups with dissimilar adsorption and assembling characteristics to the top and bottom phthalocyaninato moieties

Address correspondence to wangch@nanoctr.cn 
of a tris(phthalocyaninato) lutetium triple-decker complex $\left(\mathrm{Lu}_{2} \mathrm{Pc}_{3}\right)$, and orientation-dependent ordering of such molecules at different bias polarities has been identified [10]. In general, the molecular switching behaviors can be explained in terms of the arrangements before and after changing bias polarities. The process of molecular switching itself has rarely been mentioned explicitly up to now, and deserves further investigation.

Scanning tunneling microscopy (STM) and spectroscopy (STS) methods enable complementary investigations of the molecular structures and electronic properties at the single molecule level by virtue of their unique ultrahigh spatial resolution and versatility. Many efforts have been made to study the electronic structure and properties of adsorbed organic molecules using STM/STS techniques [11-19]. Bias-dependent visualization of molecules containing both electron-donor and -acceptor moieties has been demonstrated [17-19], which reveals the sensitivity of STM to the electronic properties of the adsorbed molecules.

In this work, the molecular switching behavior of the asymmetric triple-decker sandwich complex $\mathrm{Lu}_{2} \mathrm{Pc}_{3}$ at the liquid/solid interface has been studied by STM and STS methods, and changes of tunneling current during the switching process have been tracked by using $\mathrm{d} I / \mathrm{d} V$ versus $V$ curves. STM observations revealed that the orientation and ordering of the adsorbed $\mathrm{Lu}_{2} \mathrm{Pc}_{3}$ molecules could be readily tuned by changing bias polarities. STS results showed a current jump which was furthermore confirmed to be related to an appreciable biasinduced adsorption reversal process for individual $\mathrm{Lu}_{2} \mathrm{Pc}_{3}$ molecules.

\section{Experimental}

\subsection{STM characterization}

The sandwich complex $\mathrm{Lu}_{2} \mathrm{Pc}_{3}$ was synthesized by the reported method and characterized by ${ }^{1} \mathrm{H}$ NMR spectroscopy and mass spectroscopy [2023]. For surface assembly studies, the complex was dissolved in 1-phenyloctane with a concentration of about $1 \mathrm{mg} / \mathrm{mL}$ and the solution $(1 \mu \mathrm{L})$ was drop- cast on a freshly cleaved highly oriented pyrolytic graphite (HOPG) surface. STM characterization of the assembled film was carried out with a Nanoscope IIIa instrument (Veeco Metrology, USA) on a 1-phenyloctane/graphite interface. The STM tips were mechanically formed $\mathrm{Pt} / \mathrm{Ir}$ wires $(80 / 20)$. The bias was applied to the substrate with the tip grounded during STM measurements.

\subsection{STS characterization}

During STS investigation, the tip was located on top of the center of a $\mathrm{Lu}_{2} \mathrm{Pc}_{3}$ molecule while feedback was turned off. Spectroscopy was performed by adding a dithering modulation (peak-to-peak 20-30 mV) to the bias voltage, and the bias was scanned through the designated voltage range. A lock-in amplifier was used to collect the $\mathrm{d} I / \mathrm{d} V$ signal. To ensure reliability and reproducibility, the spectra were averaged over a large number of characteristic curves on different $\mathrm{Lu}_{2} \mathrm{Pc}_{3}$ molecules in different regions.

\subsection{Computational details}

We performed theoretical calculations using density functional theory (DFT) provided by the DMol3 code [24]. The Perdew and Wang parameterization [25] of the local exchange-correlation energy was applied for the local spin density approximation (LSDA) to describe exchange and correlation. We expanded the all-electron spin-unrestricted Kohn-Sham wave functions in a local atomic orbital basis. A doublenumerical basis set polarization was employed. All calculations were of the all-electron type, and performed with the extra-fine mesh.

\section{Results and discussion}

The family of phthalocyanines (Pcs) represents one of the promising candidates for forming ordered thin films in organic electronics due to their significant chemical stability and electronic properties [26-28]. Because of the intriguing inter-ring interactions and the intrinsic nature of the metal centers, sandwichtype double- and triple-decker complexes of Pcs display characteristic features, which are different from their non-sandwich counterparts, enabling their applications in different areas such as electrochromic 


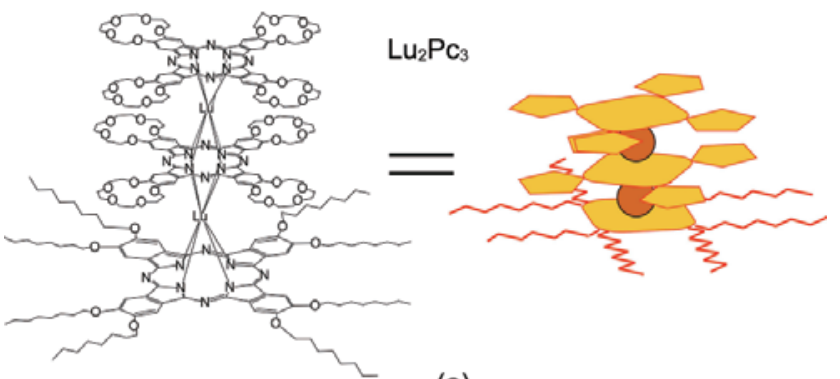

(a)

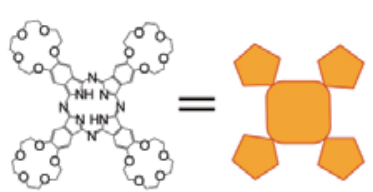

(b)

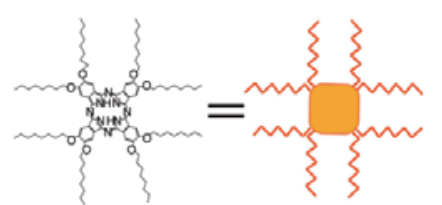

(c)
Scheme 1 (a) Molecular structure of the noncentrosymmetric tris(phthalocyaninato) lutetium triple-decker complex $\left(\mathrm{Lu}_{2} \mathrm{PC}_{3}\right)$; (b) 15-crown-5 substituted Pc (Pc[15C5] $)_{4}$ moiety; (c) $2,3,9,10,16,17,23,24$-octakis(octyloxy)-phthalocyanine (PcOC8) moiety. The orange figures illustrate geometric representations of the structures

displays, field-effect transistors and gas sensors [2931].

The chemical structure of the triple-decker complex $\mathrm{Lu}_{2} \mathrm{Pc}_{3}$ is shown in Scheme 1 (a). $\mathrm{Lu}_{2} \mathrm{Pc}_{3}$ is cylindrical in apparent shape with its height reaching nearly $1 \mathrm{~nm}$, comparable to the lateral size of the phthalocyanine ring $(1.3 \mathrm{~nm})$. In comparison with their monophthalocyanine counterparts, the assembly of sandwich complexes is more challenging due to their non-planar characteristics [32-36]. The sensitive response of the dipole to an electric field is expected to influence the adsorption of the tripledecker complexes.

As described previously, we use a strategy of attaching substituents of different polarity to the top and bottom phthalocyaninato moieties to generate an intrinsic molecular dipole [10]. Due to the high electronegativity of the oxygen atoms, the attachment of 15-crown-5 moieties to the Pc ring is expected to cause charge separation in the molecule, where the upper 15-crown-5 substituted $\mathrm{Pc}\left(\mathrm{Pc}_{\mathrm{C}}[15 \mathrm{C} 5]_{4}\right)$ moiety (Scheme 1(b)) is negatively charged and the lower 2,3,9,10,16,17,23,24-octakis(octyloxy)-phthalocyanine (PcOC8) one (Scheme 1(c)) is positively charged. Theoretical calculations using DFT provided by the DMo13 code revealed a large intrinsic molecular dipole reaching $17.5 \mathrm{D}$ along the axial direction of
$\mathrm{Lu}_{2} \mathrm{Pc}_{3}[24,25]$. It is expected that the positively polarized PcOC 8 moiety will face toward the substrate when the sample bias is negative, and the negatively polarized $\mathrm{Pc}[15 \mathrm{C} 5]_{4}$ will face to the surface when positive bias is applied.

Our STM observations, similar to those reported earlier [10], confirmed the above conjecture. They revealed that $\mathrm{Lu}_{2} \mathrm{Pc}_{3}$ molecules packed with different symmetries according to the applied electric field between the STM tip and the substrate. Large areas of ordered assembled structures with fourfold symmetries were observed for $\mathrm{Lu}_{2} \mathrm{Pc}_{3}$, molecules at the 1-phenyloctane/graphite interface when the applied sample bias was varied from -300 to $-1500 \mathrm{mV}$ (Fig. 1(a)). The intermolecular distance estimated from the STM image is about $2.6 \mathrm{~nm}$, in good agreement with the earlier value for a monophthalocyanine CuPcOC8 assembly $[37,38]$, indicating that $\mathrm{Lu}_{2} \mathrm{Pc}_{3}$ has adopted a face-on adsorption configuration with the PcOC8 moiety facing towards the graphite surface and the octyloxy groups fully interdigitated with those of the neighboring molecules. In contrast, a disordered assembly was observed at the interface when a positive bias was applied, as shown in Fig. 1(b). Due to the lack of long range ordering, precise determination of the intermolecular distance was difficult in this assembly. In the disordered assembled structure the molecule can be considered to be adsorbed with the $\mathrm{Pc}[15 \mathrm{C} 5]_{4}$ moiety oriented towards the surface, with $\mathrm{PcOC} 8$ pointing away from the

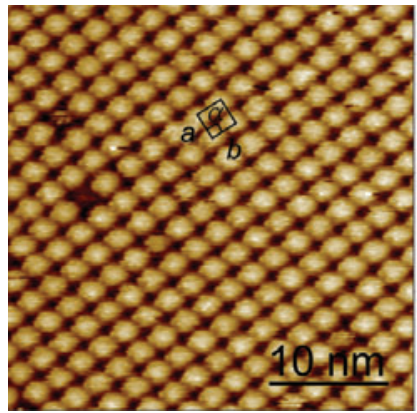

(a)

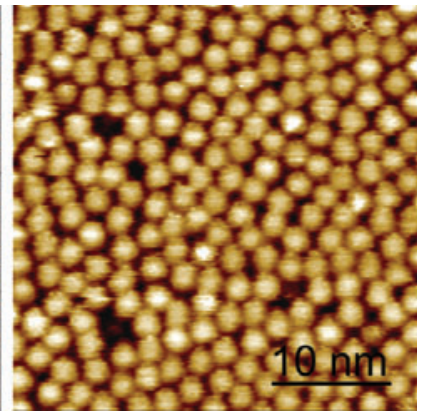

(b)
Figure 1 Typical STM images for the assembly of $\mathrm{Lu}_{2} \mathrm{PC}_{3}$ tripledecker complexes at the phenyloctane/graphite interface revealed under opposite bias polarities. Tunneling conditions: (a) $-1.0 \mathrm{~V}$, 315 pA; (b) 1.5 V, 315 pA. (a) Packing arrangements with fourfold symmetries under negative sample bias. Unit cell parameters: $a=2.6 \mathrm{~nm} \pm 0.1 \mathrm{~nm}, b=2.6 \mathrm{~nm} \pm 0.1 \mathrm{~nm}, \alpha=90^{\circ} \pm 1.0^{\circ}$; (b) disordered monolayer structure under positive sample bias 
substrate towards the solution.

By changing bias polarity, the assembled structures of $\mathrm{Lu}_{2} \mathrm{Pc}_{3}$ could be reversibly switched between ordered and disordered. A typical switching process is shown in Fig. 2. Initially an ordered monolayer of $\mathrm{Lu}_{2} \mathrm{Pc}_{3}$ molecules was revealed under negative bias (Fig. 2(a)). As the bias polarity was suddenly changed to positive, deconstruction of the ordered domain was immediately induced (Fig. 2(b)), and the assembled layer gradually became disordered (after around 1 min) (Fig. 2(c)). The locations of the $\mathrm{Lu}_{2} \mathrm{Pc}_{3}$ molecules in Fig. 2(c) are similar, but not identical, to those in Fig. 2(b). This suggests that although the molecules could not return to their original location after switching, they tended to stay as near as possible to their previous locations. When the bias was changed

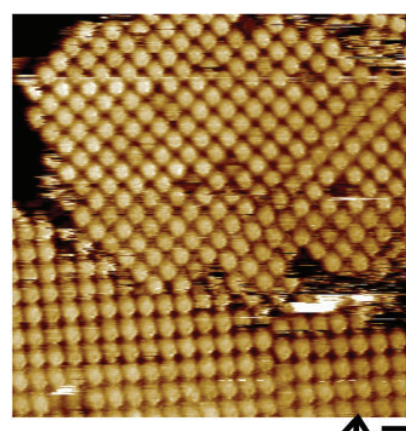

(a)

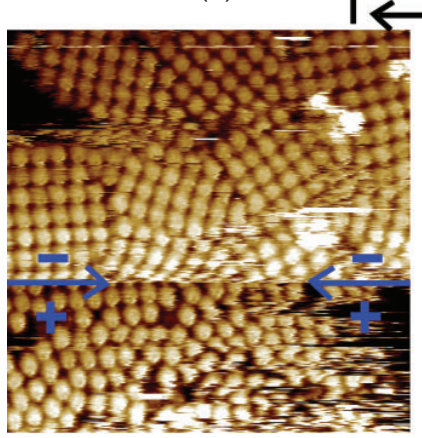

(d)



(b)

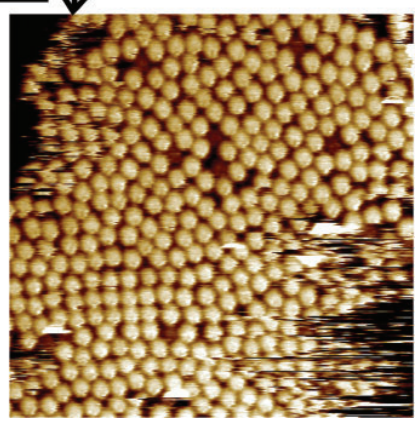

(c)

Figure 2 Sequential STM images obtained for the same area illustrating the bias-polarity-induced phase transition. At the beginning (a), bias is set to $-970 \mathrm{mV}$ (the tunneling current is maintained 363 pA through out the process). At the place marked by the blue arrows in image (b), the bias was suddenly changed to $970 \mathrm{mV}$, which immediately induced deconstruction of the ordered domain and the assembled layer finally becomes disordered lacking long range order after $1 \mathrm{~min}$ (c). In image (d) the bias has been changed back to $-970 \mathrm{mV}$ at the place marked by the blue arrows. This change of sample bias immediately induced a phase transition from disordered to ordered in the assembled layer. During the scanning under this negative bias, a consecutive growth of the fourfold ordered domains could be observed (d) to (a) from positive back to negative, an immediate phase transition occurred and the disordered assembly reverted to an ordered one (Fig. 2(d)).

The most intriguing result was that some abrupt peaks appeared in the $\mathrm{d} I / \mathrm{d} V$ spectrum when the STS method was employed to estimate the electronic properties of the adsorbed $\mathrm{Lu}_{2} \mathrm{Pc}_{3}$ molecules. Figure 3 shows the typical $\mathrm{d} I / \mathrm{d} V$ versus $V$ curves obtained by locating the STM tip on top of individual $\mathrm{Lu}_{2} \mathrm{Pc}_{3}$ molecules. The spectrum of $\mathrm{Lu}_{2} \mathrm{Pc}_{3}$ shows a characteristic energy gap, revealing probable semiconductor behaviour. It should be noted that the experimentally determined apparent energy gap (about $2.4 \mathrm{eV}$ ) obtained from STS results is somewhat wider than those of single-decker metallophthalocyanines ( $\mathrm{MPc})$, such as copper phthalocyanine [39] and titanyl phthalocyanine [40] whose apparent gaps are about $2 \mathrm{eV}$. The value of the apparent gap for $\mathrm{Lu}_{2} \mathrm{Pc}_{3}$ is also different from the results obtained from electronic adsorption

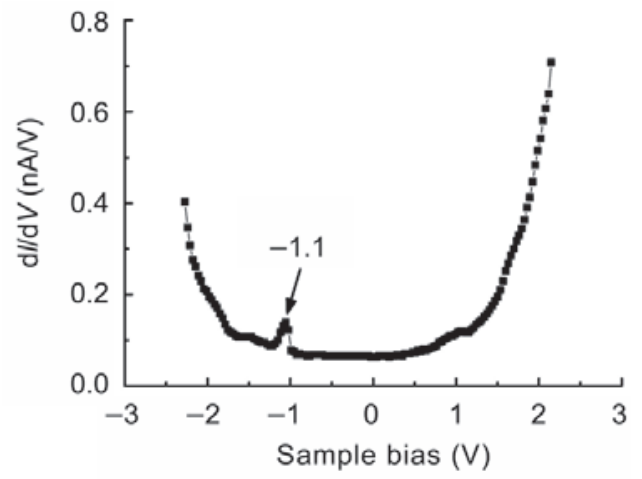

(a)

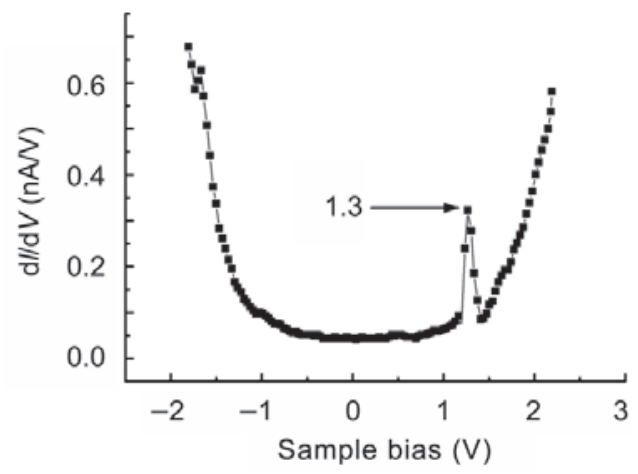

(b)

Figure 3 (a) Typical $d / / d V$ versus $V$ curves for $\mathrm{Lu}_{2} \mathrm{PC}_{3}$ molecules. (STM/ STS conditions: $-1.10 \mathrm{~V}, 300 \mathrm{pA}$ ). An abrupt peak appeared around $-1.1 \mathrm{~V}$ when the bias was scanned from $2.5 \mathrm{~V}$ to $-2.5 \mathrm{~V}$, while the peak appeared around $1.3 \mathrm{~V}$ when the bias was scanned from negative to positive 
spectroscopy and cyclic voltammetry, which give a smaller gap for triple-decker complexes, ascribed to intrinsic face-to-face stacking and strong interactions between the phthalocyanine rings [41-44]. Here, we would like to focus on the abrupt jumps in the $\mathrm{d} I / \mathrm{d} V$ spectra during the bias scanning through the designated voltage range, which has never been reported in STS observations of single-decker MPC molecules [11, 12, 39, 40]. Abrupt peaks appeared around $-1.1 \mathrm{~V}$ when the bias was changed from 2.5 $\mathrm{V}$ to $-2.5 \mathrm{~V}$, while the peak shifted to around $1.3 \mathrm{~V}$ when the bias was scanned from negative to positive; the results exhibited good reproducibility, which excludes random fluctuations or noise as possible explanations.

The abrupt peaks in the $\mathrm{d} I / \mathrm{d} V$ spectra of the $\mathrm{Lu}_{2} \mathrm{Pc}_{3}$ triple-decker complex could be associated with the flipping process of $\mathrm{Lu}_{2} \mathrm{Pc}_{3}$ on HOPG induced by changing of the bias polarity. For molecular flipping, a desorption process from the surface is necessary, which would reduce the adsorbate-substrate interactions and might affect the electron tunneling behaviour; we suggest this as a possible reason for the abrupt jumps in tunneling current observed in the STS spectra. When the bias was changed from positive to negative, $\mathrm{Pc}[15 \mathrm{C} 5]_{4}$ moieties could be destabilized from the surface driven by the external electric field. The solid/liquid interface provides a free space for $\mathrm{Lu}_{2} \mathrm{Pc}_{3}$ molecules to flip. Due to the high affinity of the octoxyl groups for the graphite surface and the strong interactions induced by octoxyl interdigitation, the other assembled structure is expected to be rather more stable and require more energy (or higher bias voltage) to destabilize the molecules when the bias is scanned from negative to positive. The above STS results combined with STM observations further confirm that the asymmetric triple-decker complex $\mathrm{Lu}_{2} \mathrm{Pc}_{3}$ can act as a molecular switch when stimulated by an electric field.

\section{Conclusions}

In summary, attaching substituents of different polarities can introduce the capability of controlling the adsorption configuration of organic molecules at interfaces by an external electric field. The as- prepared asymmetric triple-decker complex $\mathrm{Lu}_{2} \mathrm{Pc}_{3}$ had a strong intrinsic molecular dipole and packed in different ways according to the applied electric field between the STM tip and substrate. The STM observations combined with STS results revealed that $\mathrm{Lu}_{2} \mathrm{Pc}_{3}$ molecules can act as a molecular switch by exhibiting a voltage induced flip at the solid/ liquid interface. Our results provide a possible way to precisely control the ordering and orientation of organic species by means of external stimuli.

\section{Acknowledgements}

The authors are grateful for financial support from the National Natural Science Foundation of China (90406019, 20573116, and 20673029) and the National Basic Research Program of China (973 Program, 2006CB932100).

\section{References}

[1] Alemani, M.; Peters, M. V.; Hecht, S.; Rieder, K. -H.; Moresco, F.; Grill, L. Electric field-induced isomerization of azobenzene by STM. J. Am. Chem. Soc. 2006, 128, 14446-14447.

[2] Yoshimoto, S.; Yokoo, N.; Fukuda, T.; Kobayashi, N.; Itaya, K. Formation of highly ordered porphyrin adlayers induced by electrochemical potential modulation. Chem. Commun. 2006, 500-502.

[3] Yang, Y. L.; Chan, Q. L.; Ma, X. J.; Deng, K.; Shen, Y. T.; Feng, X. Z.; Wang, C. Electrical conformational bistability of dimesogen molecules with a molecular chord structure. Angew. Chem., Int. Ed. 2006, 45, 6889-6893.

[4] Bondos, J. C.; Drummer, N. E.; Gewirth, A. A.; Nuzzo, R. G. Thermal phase evolution of Pt-Si intermetallic thin films prepared by the activated adsorption of $\mathrm{SiH}_{4}$ on $\mathrm{Pt}(100)$ and comparison to known structural models. J. Am. Chem. Soc. 1999, 121, 2498-2507.

[5] Ruben, M.; Payer, D.; Landa, A.; Comisso, A.; Gattinoni, C.; Lin, N.; Collin, J. -P.; Sauvage, J. -P.; De Vita, A.; Kern, K. 2-D supramolecular assemblies of benzene1,3,5-triyl-tribenzoic acid: Temperature-induced phase transformations and hierarchical organization with macrocyclic molecules. J. Am. Chem. Soc. 2006, 128, 15644-15651

[6] Rohde, D.; Yan, C. J.; Yan, H. J.; Wan, L. J. From a 
lamellar to hexagonal self-assembly of bis $\left(4,4^{\prime}-\left(m, m^{\prime}-\right.\right.$ di(dodecyloxy)phenyl)-2,2'-difluoro-1,3,2-dioxaborin) molecules: A trans-to-cis-isomerization-induced structural transition studied with STM. Angew. Chem. Int. Ed. 2006, 45, 3996-4000.

[7] Kong, X. H.; Deng, K.; Yang, Y. L.; Zeng, Q. D.; Wang, $C$. Effect of thermal annealing on hydrogen bond configurations of host lattice revealed in VOPC/TCDB host-guest architectures. J. Phys. Chem. C 2007, 111, 9235-9239.

[8] Bissell, R. A.; Cordova, E.; Kaifer, A. E.; Stoddart, J. F. A chemically and electrochemically switchable molecular shuttle. Nature 1994, 369, 133-137.

[9] Gopakumar, T. G.; Müller, F.; Hietschold, M. Scanning tunneling microscopy and scanning tunneling spectroscopy studies of planar and nonplanar naphthalocyanines on graphite (0001). Part 1: Effect of nonplanarity on the adlayer structure and voltageinduced flipping of nonplanar tin-naphthalocyanine. J. Phys. Chem. B 2006, 110, 6051-6059.

[10] Lei, S. B.; Deng, K.; Yang, Y. L.; Zeng, Q. D.; Wang, C.; Jiang, J. Z. Electric driven molecular switching of asymmetric tris(phthalocyaninato) lutetium triple-decker complex at the liquid/solid interface. Nano Lett. 2008, 8, 1836-1843.

[11] Qiu, X. H.; Nazin, G. V.; Ho, W. Vibrationally resolved fluorescence excited with submolecular precision. Science 2003, 299, 542-546.

[12] Nazin, G. V.; Qiu, X. H.; Ho. W. Visualization and spectroscopy of a metal-molecule-metal bridge. Science 2003, 302, 77-81.

[13] Scudiero, L.; Barlow, D. E.; Mazur, U.; Hipps, K. W. Scanning tunneling microscopy, orbital-mediated tunneling spectroscopy, and ultraviolet photoelectron spectroscopy of metal(II) tetraphenylporphyrins deposited from vapor. J. Am. Chem. Soc. 2001, 123, 4073-4080.

[14] Deng, W.; Hipps, K. W. Tip-sample distance dependence in the STM-based orbital-mediated tunneling spectrum of nickel(II) tetraphenylporphyrin deposited on Au(111). J. Phys. Chem. B 2003, 107, 10736-10740.

[15] Ouyang, M.; Huang, J. L.; Cheung, C. L.; Lieber, C. M. Energy gaps in "metallic" single-walled carbon nanotubes. Science 2001, 292, 702-705.

[16] Cui, X. D.; Primak, A.; Zarate, X.; Tomfohr, J.; Sankey, O. F.; Moore, A. L.; Moore, T. A.; Gust, D.; Harris, G.; Lindsay, S. M. Reproducible measurement of single- molecule conductivity. Science 2001, 294, 571-574.

[17] Jäckel, F.; Yin, X.; Samorì, P.; Tchebotareva, N.; Watson, M. D.; Venturini, A.; Müllen, K.; Rabe, J. P. Nanophase segregation and rectification in monolayers of functionalized hexa peri-hexabenzocoronenes. Synth. Met. 2004, 147, 5-9.

[18] Samorì, P.; Yin, X.; Tchebotareva, N.; Wang, Z.; Pakula, T.; Jäfckel, F.; Watson, M. D.; Venturini, A.; Müllen, K.; Rabe, J. P. Self-assembly of electron donor-acceptor dyads into ordered architectures in two and three dimensions: Surface patterning and columnar "double cables". J. Am. Chem. Soc. 2004, 126, 3567-3575.

[19] Miura, A.; Chen, Z.; Uji-i, H.; De Feyter, S.; Zdanowska, M.; Jonkheijm, P.; Schenning, A. P. H. J.; Meijer, E. W.; Würthner, F.; De Schryver, F. C. Bias-dependent visualization of electron donor (D) and electron acceptor (A) moieties in a chiral DAD triad molecule. J. Am. Chem. Soc. 2003, 125, 14968-14969.

[20] Jiang, J.; Bian, Y.; Furuya, F.; Liu, W.; Choi, M. T. M.; Kobayashi, N.; Li, H. W.; Yang, Q.; Mak, T. C. W.; Ng, D. K. P. Synthesis, structure, spectroscopic properties, and electrochemistry of rare earth sandwich compounds with mixed 2,3-naphthalocyaninato and octaethylporphyrinato ligands. Chem. Eur. J. 2001, 7, 5059-5069.

[21] Zhu, P.; Pan, N.; Li, R.; Dou, J.; Zhang, Y.; Cheng, D. Y. Y.; Wang, D.; Ng, D. K. P.; Jiang, J. Electrondonating alkoxy-group-driven synthesis of heteroleptic tris(phthalocyaninato) lanthanide(III) triple-deckers with symmetrical molecular structure. Chem. Eur. J. 2005, 11, 1425-1432.

[22] Tashiro, K.; Konishi, K.; Aida, T. Metal bisporphyrinate double-decker complexes as redox-responsive rotating modules. Studies on ligand rotation activities of the reduced and oxidized forms using chirality as a probe. $J$. Am. Chem. Soc. 2000, 122, 7921-7926.

[23] Chen, Y.; Su, W.; Bai, M.; Jiang, J.; Li, X.; Liu, Y.; Wang, L.; Wang, S. High performance organic field-effect transistors based on amphiphilic tris(phthalocyaninato) rare earth triple-decker complexes. J. Am. Chem. Soc. 2005, 127, 15700-15701.

[24] Becke, A. D. A multicenter numerical integration scheme for polyatomic molecules. J. Chem. Phys. 1988, 88, 2547 $-2553$.

[25] Perdew, J. P.; Wang, Y. Accurate and simple analytic representation of the electron-gas correlation energy. 
Phys. Rev. B 1992, 45, 13244-13249.

[26] Collins, R. A.; Mohammed, K. A. Gas sensitivity of some metal phthalocyanines. J. Phys. D: Appl. Phys. 1988, 21, 154-161.

[27] Bao, Z.; Lovinger, A. J.; Dodabalapur, A. Organic fieldeffect transistors with high mobility based on copper phthalocyanine. Appl. Phys. Lett. 1996, 69, 3066-3068.

[28] Bao, Z.; Lovinger, A. J.; Dodabalapur, A. Highly ordered vacuum-deposited thin films of metallophthalocyanines and their applications in field-effect transistors. Adv. Mater. 1997, 9, 42-44.

[29] Nicholson, M. M. In Phthalocyanines - Properties and Applications, Vol. 3; Leznoff, C. C.; Lever, A. B. P., Eds.; VCH: New York, 1993, pp. 71-117.

[30] Madru, R.; Guillaud, G.; Al Sadoun, M.; Maitrot, M.; André, J. -J.; Simon, J.; Even, R. A well-behaved field-effect transistor based on an intrinsic molecular semiconductor. Chem. Phys. Lett. 1988, 145, 343-346.

[31] Rickwood, K. R.; Lovett, D. R.; Lukas, B.; Silver, J. Semiconducting, pyroelectric and chlorine-sensing properties of ytterbium bisphthalocyanine LangmuirBlodgett thin-films. J. Mater. Chem. 1995, 5, 725-729.

[32] Klymchenko, A. S.; Sleven, J.; Binnemans, K.; De Feyter, S. Two-dimensional self-assembly and phase behavior of an alkoxylated sandwich-type bisphthalocyanine and its phthalocyanine analogues at the liquid-solid interface. Langmuir 2006, 22, 723-728.

[33] Binnemans, K.; Sleven, J.; De Feyter, S.; De Schryver, F. C.; Donnio, B.; Guillon, D. Structure and mesomorphic behavior of alkoxy-substituted bis(phthalocyaninato)lan thanide(III) complexes. Chem. Mater. 2003, 15, 39303938.

[34] Yang, Z. Y.; Gan, L. H.; Lei, S. B.; Wan, L. J.; Wang, C.; Jiang, J. Z. Self-assembly of PcOC 8 and its sandwich lanthanide complex $\operatorname{Pr}(\mathrm{PcOC} 8) 2$ with oligo(phenyleneethynylene) molecules. J. Phys. Chem. B 2005, 109, 19859-19865.

[35] Ma, H.; Ou Yang, L. Y.; Pan, N.; Yau, S. L.; Jiang, J.; Itaya, K. Ordered molecular assemblies of substituted bis(phthalocyaninato) rare earth complexes on $\mathrm{Au}(111)$ : In situ scanning tunneling microscopy and electrochemical studies. Langmuir 2006, 22, 2105-2111.

[36] Takami, T. ; Arnold, D. P.; Fuchs, A. V.; Will, G. D.; Goh,
R.; Waclawik, E. R.; Bell, J. M.; Weiss, P. S.; Sugiura, K.; Liu, W.; Jiang, J. Two-dimensional crystal growth and stacking of bis(phthalocyaninato) rare earth sandwich complexes at the 1-phenyloctane/graphite interface. J. Phys. Chem. B 2006, 110, 1661-1664.

[37] Qiu, X.; Wang, C.; Zeng, Q.; Xu, B.; Yin, S.; Wang, H.; $\mathrm{Xu}, \mathrm{S}$.; Bai, C. Alkane-assisted adsorption and assembly of phthalocyanines and porphyrins. J. Am. Chem. Soc. 2000, 122, 5550-5556.

[38] Qiu, X.; Wang, C.; Yin, S.; Zeng, Q.; Xu, B.; Bai, C. Selfassembly and immobilization of metallophthalocyanines by alkyl substituents observed with scanning tunneling microscopy. J. Phys. Chem. B 2000, 104, 3570-3574.

[39] Lei, S. B.; Deng, K.; Yang, D. L.; Zeng, Q. D.; Wang, C. Charge-transfer effect at the interface of phthalocyanineelectrode contact studied by scanning tunneling spectroscopy. J. Phys. Chem. B 2006, 110, 1256-1260.

[40] Kong, X. H.; Wang, M.; Lei, S. B.; Yang, Y. L.; Wang, C. Electronic sensory behavior of titanylphthalocyanine revealed by scanning tunneling spectroscopy and cyclic voltammetry methods. J. Mater. Chem. 2006, 16, 42654269.

[41] Ishikawa, N. Electronic structures and spectral properties of double- and triple-decker phthalocyanine complexes in a localized molecular orbital view. J. Porphyr. Phthalocya. 2001, 5, 87-101.

[42] Chen, Y. L.; Li, R. J.; Wang, R. M.; Ma, P.; Dong, S.; Gao, Y. N.; Li, X. Y.; Jiang, J. Z. Effect of peripheral hydrophobic alkoxy substitution on the organic field effect transistor performance of amphiphilic tris(phthalocyaninato) europium triple-decker complexes. Langmuir 2007, 23, 12549-12554.

[43] Li, R. J.; Ma, P.; Dong, S.; Zhang, X. Y.; Chen, Y. L.; Li, X. Y.; Jiang, J. Z. Synthesis, characterization, and OFET properties of amphiphilic heteroleptic tris(phthalocyaninato) europium(III) complexes with hydrophilic poly(oxyethylene) substituents. Inorg. Chem. 2007, 46, 11397-11404.

[44] Guyon, F.; Pondaven, A.; Kerbaol, J. -M.; L'Her, M. From the single-to the triple-decker sandwich. Effect of stacking on the redox and UV-visible spectroscopic properties of lutetium(III) 1,2-naphthalocyaninate complexes. Inorg. Chem. 1998, 37, 569-576. 